\title{
Optimization of evanescent wave imaging for the visualization of protein adsorption layers
}

\author{
LIU Li ${ }^{1,2}$, NIU Yu ${ }^{1,2}$, CHEN She ${ }^{1,2}$, MENG YongHong ${ }^{1,2}$, MA HongWei $^{3} \&$ JIN Gang $^{1 *}$ \\ ${ }^{1}$ Department of National Microgravity Laboratory, Institute of Mechanics, Chinese Academy of Sciences, Beijing 100190, China; \\ ${ }^{2}$ Graduate University of Chinese Academy of Sciences, Beijing 100049, China; \\ ${ }^{3}$ Institute of Nano-tech and Nano-bionics, Chinese Academy of Sciences, Suzhou 215125, China
}

Received February 27, 2010; accepted May 18, 2010; published online August 25, 2010

\begin{abstract}
We have optimized the settings of evanescent wave imaging for the visualization of a protein adsorption layer. The enhancement of the evanescent wave at the interface brought by the incident angle, the polarized state of light beam as well as a gold layer is considered. In order to improve the image contrast of a protein monolayer in experiments, we have optimized three factors - the incident angle, the polarization of light beam, and the thickness of an introduced thin gold layer with a theoretical simulation.
\end{abstract}

optimization, evanescent wave imaging, image contrast

PACS: 07.05.Pj, 07.60.-j, 78.20.Bh

\section{Introduction}

Evanescent wave arises at the interface of two media when light propagates from a dense medium to a rare one in the total internal reflection condition, which is distributed in a superficial area since its amplitude decays exponentially with the distance less than the wavelength from the interface [1] and the intensity of the evanescent wave at the interface can be larger than that of the input beam [2]. In light of its localization and amplitude enhancement, the evanescent wave has been used in the imaging of chemical, bio-chemical and biological phenomena on the interface. For example, the evanescent wave is responsible for the excitation of the fluorophore in total internal reflection fluorescence microscopy (TIRFM) [3], which is used to visualize single-molecules on the surface of living cells [4], and detect cell membranes in micro-domains [5]. In addition, based on the principle of surface plasmon resonance,

*Corresponding author (email: gajin@imech.ac.cn) the SPR imaging biosensor is applied in many fields such as antibody-antigen binding [6], DNA hybridization [7], and protein-DNA interactions [8]. But a problem still in the way is how to improve the image contrast of a monolayer. In this paper, we visualize a protein adsorption layer with evanescent wave imaging and study the possibility, experimental conditions and contributions of evanescent wave imaging in a theoretical simulation and experiments. The imaging system for the visualization of the protein layer is shown in Figure 1 and the zoom in Figure 1 is the physical model of sample for the simulation.

\section{Theoretical simulation}

For the theoretical simulation, we consider that a light beam illuminates a stratified structure-a stack of $0 \ldots n(1 \leqslant n \leqslant 3$ integer) parallel layers sandwiched between the ambient $(0)$ and the substrate $(n)$ shown in the zoom of Figure 1. The " $n$ " is the medium number of the stratified structure in the physical model of the sample. Suppose all the media were 


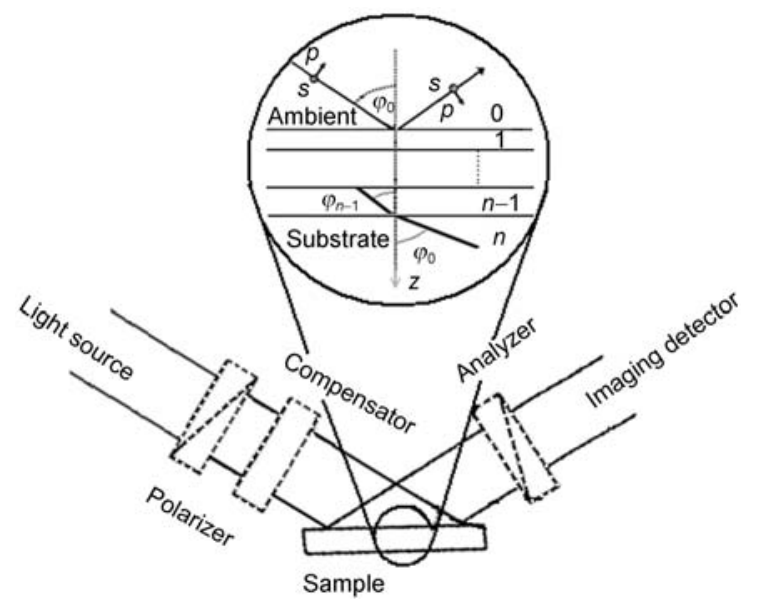

Figure 1 The schematic diagram of the evanescent wave imaging system. The zoom is the physical model of the sample.

homogeneous and isotropic. The simulation is described with: (1) Maxwell's field equations are used in isotropic media; (2) Suppose the ambient medium, the individual layer or layers, and the substrate were infinite planar; (3) The light intensity at both sides of an interface is continuous.

For simplification, the amplitude of the incident wave is set in a unit. The incident light wave is described with both polarization components of parallel $(p)$ and perpendicular $(s)$ to the plane of incidence. We set $N_{i}$ and $d_{i}(0 \leqslant i \leqslant n)$ as the complex indices of refraction and the thickness of the $i$ th layer. We apply the Snell's law as:

$$
N_{0} \sin \varphi_{0}=N_{1} \sin \varphi_{1}=\cdots=N_{i} \sin \varphi_{i}=\cdots=N_{n} \sin \varphi_{n} .
$$

The Fresnel reflection $(r)$ and transmission $(t)$ coefficients in the $p$ and $s$ direction at an individual interface are shown as:

$$
\begin{aligned}
& r_{(n-1, n) p}=\frac{N_{n} \cos \varphi_{n-1}-N_{n-1} \cos \varphi_{n}}{N_{n} \cos \varphi_{n-1}+N_{n} \cos \varphi_{n-1}} \\
& r_{(n-1, n) s}=\frac{N_{n-1} \cos \varphi_{n-1}-N_{n} \cos \varphi_{n}}{N_{n-1} \cos \varphi_{n-1}+N_{n} \cos \varphi_{n}}, \\
& t_{(n-1, n) p}=\frac{2 N_{n-1} \cos \varphi_{n-1}}{N_{n} \cos \varphi_{n-1}+N_{n-1} \cos \varphi_{n}}, \\
& t_{(n-1, n) s}=\frac{2 N_{n-1} \cos \varphi_{n-1}}{N_{n-1} \cos \varphi_{n-1}+N_{n} \cos \varphi_{n}} .
\end{aligned}
$$

The complex-amplitude reflection $(R)$ and transmission $(T)$ can be deduced by the addition of an infinite geometric series consisting of the reflection $(r)$ or transmission $(t)$ coefficients and the phase change $\beta$ at an individual interface.

$$
\beta=\frac{2 \pi d_{i} N_{i}}{\lambda} \cos \varphi_{i}
$$

$I_{p}$ and $I_{s}$ are the $p$ and $s$ component intensity, which is given by

$$
\begin{array}{cc}
I_{r p}=\left|R_{p}\right|^{2}, & I_{r s}=\left|R_{s}\right|^{2}, \\
I_{t p}=\left|T_{p}\right|^{2}, & I_{t s}=\left|T_{s}\right|^{2} .
\end{array}
$$

Equations could be obtained in Azzam and Bashara's publication [9].

A light beam goes from glass to liquid. Suppose the refractive index of the glass and the liquid were 1.72 and 1.33 at the wavelength of $633 \mathrm{~nm}$. The evanescent wave appears at the side of liquid on the interface of two media when the angle of incidence is larger than the critical angle [10]. The relationship of the evanescent wave intensity and the incident angle is simulated in Figure 2 which shows a sharp increase at the incident angle reaching the critical angle [11], and the enhanced intensity of the evanescent wave is clearly near 8 times of the incident intensity.

\section{Image contrast of the protein layer on glass}

Our goal is to visualize a protein layer on a solid substrate, so we define an image contrast $\Delta I$ which is a contrast between the protein layer and the substrate. The reflection intensity of the substrate and the $d_{p r}$ thick protein layer are $I_{r}$ $=I\left(\varphi_{0}, 0\right)$ and $I_{r}=I\left(\varphi_{0}, d_{p r}\right)$, respectively, so that the image contrast could be described as $\Delta I=\frac{I\left(\varphi_{0}, d_{p r}\right)-I\left(\varphi_{0}, 0\right)}{I_{i}}$.

Here, $I_{i}$ is the intensity of the input light. In this paper, the light source is the same so that $I_{i}$ is the same in different situations and the image contrast is decided by the reflection intensity of the substrate and the protein layer. The refractive index of the protein layer was assumed to be $N_{2}=1.46$ upon the previous work [12]. The image contrast of a $2 \mathrm{~nm}$ thick protein layer is simulated in Figure 3. The absolute value of the maximum contrast for the protein adsorption

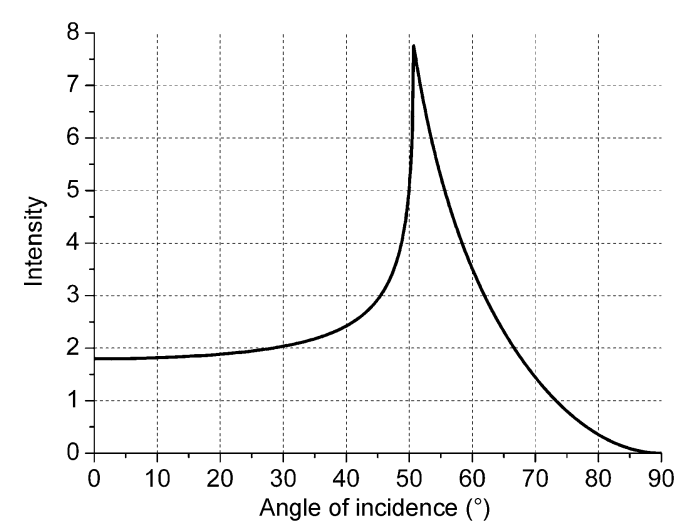

Figure 2 The intensity of evanescent wave at the glass/liquid interface vs. the angle of incidence. The refractive indices of the glass and the liquid are supposed to be 1.72 and 1.33 at $633 \mathrm{~nm}$, respectively. 


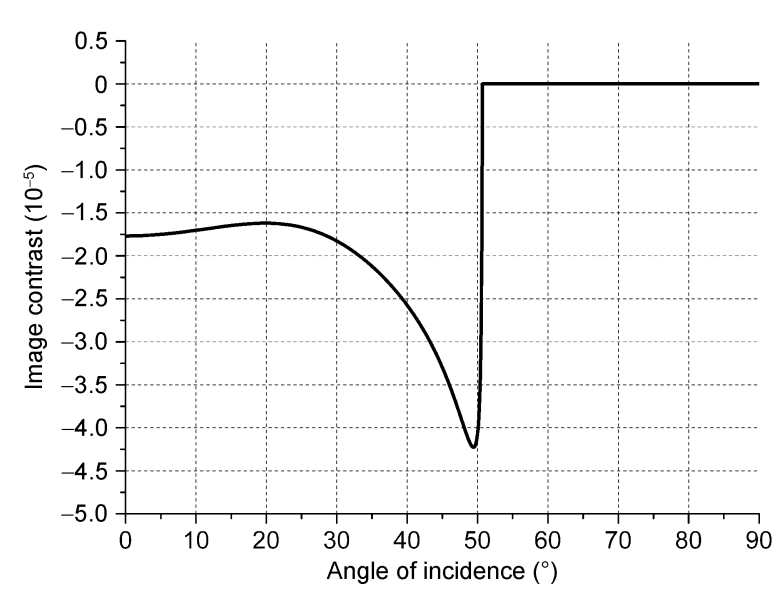

Figure 3 The image contrast of a $2 \mathrm{~nm}$ thick protein layer on glass vs. the angle of incidence. The refractive indices of the glass, the protein layer, and the liquid are supposed to be $1.72,1.46$, and 1.33 at $633 \mathrm{~nm}$, respectively.

layer on the glass/liquid interface is $4 \times 10^{-5}$ at the critical angle $50.7^{\circ}$, so that the protein layer is hardly visualized. The image contrast has to be further increased.

\section{Effect of polarization on image contrast}

The imaging system of evanescent wave to visualize a protein layer is one kind of oblique imaging. The polarization state of light would change when the light interacts with the sample, so we study the evolution of the polarization state of light for the image contrast. Each polarized light wave could be described with its amplitude and phase. The protein layer usually could be seen as a phase object which almost does not bring any change in the amplitude of probe light, but result in some change in the phase [13]. We expect that the polarization setting of light beam would improve the image contrast of the protein layer.

We introduce a conventional polarization set with polarized elements of polarizer $P$, compensator $C$, sample $S$ and analyzer $A$ for the imaging (PCSA). The intensity of the polarized imaging system is expressed as follows:

$$
\begin{aligned}
I= & K\left(\left|R_{s}\right|^{2} / 2\right)\left\{[1+\cos 2 C \cos 2(P-C)] \cos ^{2} A \tan ^{2} \psi\right. \\
& +[1-\cos 2 C \cos 2(P-C)] \sin ^{2} A \\
& +[\sin 2 C \cos 2(P-C) \cos \Delta-\sin 2(P-C) \sin \Delta] \\
& \times \sin 2 A \tan \psi\} .
\end{aligned}
$$

In eq. (7), $K$ is a compound coefficient decided by the light source and the detector which is set as 1 for accordance.

$$
\rho=\frac{R_{p}}{R_{s}}=\tan \psi \exp (\mathrm{i} \Delta)
$$

$R_{p}$ and $R_{s}$ are the Fresnel reflection coefficients which could be deduced from eqs. (1)-(4). $\psi$ and $\Delta$ are the so-called ellipsometric angles which depend on the morphology and the composition of the sample system. Only $P$ and $A$ are discussed since any value of $P-C$ can be obtained by varying $P$ at a fixed $C$ [14], and here $C$ is fixed at $45^{\circ}$ for a general consideration. The image contrast is $\Delta I=I\left(\varphi_{0}, P, A, d_{p r}\right)$ $-I\left(\varphi_{0}, P, A, 0\right)$ which is a function of the incident angle and the azimuths setting of $A, P$. We are discussing a $2 \mathrm{~nm}$ thick protein layer on a glass substrate in a simulation. In Figure 4 the maximum image contrast is obtained at the incident angle of $50.7^{\circ}$ while $A=135^{\circ}, P=0^{\circ}$ and $A=45^{\circ}, P=90^{\circ}$. The maximum image contrast of the protein layer is about $4 \times 10^{-3}$. It's obvious that the optimized polarization settings make the maximum image contrast increase about $10^{2}$ times. The image contrast is still not large enough for the visualization of the protein layer, so the signal enhancement should be considered further.

\section{Surface enhancement effect on image contrast}

Numerous researchers discover that some metal layers (gold, silver etc.) have surface enhancement effect in physical measurements. The incident wave can propagate into the metal layer and excite electromagnetic waves in metal [15]. We introduce a gold layer on the glass substrate to discover the signal enhancement possibility for the evanescent wave imaging. The intensity of the evanescent wave on the metal layer surface depends on the angle of incidence $\varphi_{0}$ and the thickness of the metal $d_{g}$. For example, we simulate the evanescent wave in the glass/gold-layer/liquid system. The gold layer with the refractive index of $N_{1}=0.19+3.10 \mathrm{j}$ is sandwiched between the glass ambient and the liquid medium. From the theoretical deduction, the intensity of the evanescent wave at the interface of gold/liquid $I_{t}=I\left(\varphi_{0}, d_{g}\right)$ is shown in Figure 5.

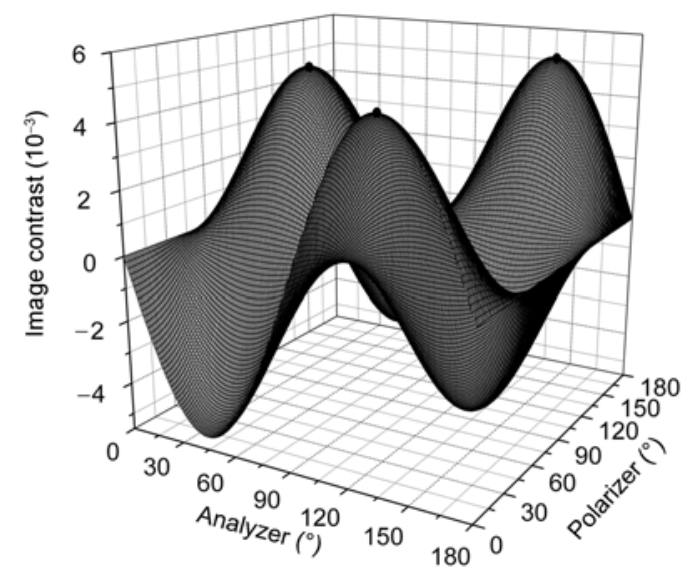

Figure 4 The image contrast vs. the azimuth setting of the polarizer and the analyzer from $0^{\circ}$ to $180^{\circ}$ in the PCSA imaging system. The sample is glass/protein adsorption layer/liquid. The maximum imaging contrast is $5.2 \times 10^{-3}$ with the optimized polarization settings of $A=135^{\circ}, P=0^{\circ}$ and $A=45^{\circ}, P=90^{\circ}$. 


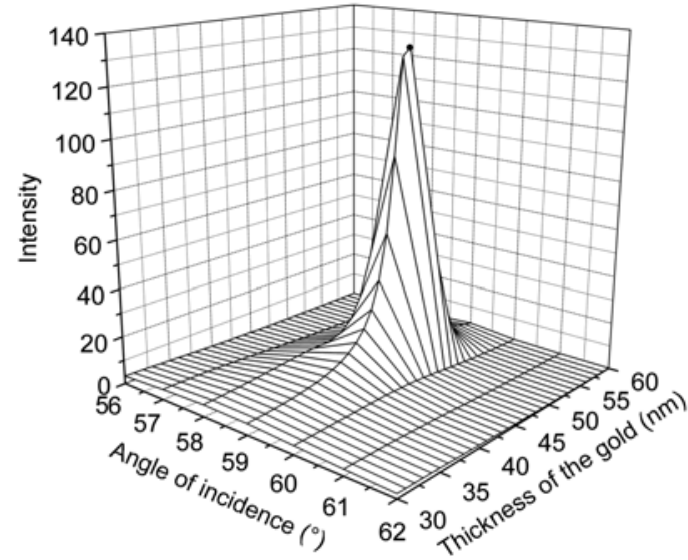

Figure 5 The intensity of the evanescent wave at the gold/liquid interface of glass/gold layer/liquid. The refractive indices of the glass, the gold and the liquid are $1.72,0.19+3.10 \mathrm{j}$, and 1.33 at $633 \mathrm{~nm}$, respectively.

The peak intensity of evanescent wave shown in Figure 5 is 128.8 when the angle of incidence is $59^{\circ}$, and the thickness of gold layer is $50 \mathrm{~nm}$. Notice that the intensity at the interface of gold-layer/liquid at the angle of incidence $59^{\circ}$ is about 17 times higher than the one at the interface of glass/liquid and the surface enhancement effect of the gold layer for the intensity of evanescent wave is very large. The intensity of the evanescent wave goes down sharply when the thickness become more intense almost 0 at the thickness $90 \mathrm{~nm}$.

Here, we would like to consider the image contrast $\Delta I$ of the protein layer at the interface of gold-layer/liquid interface. The contrast depends upon the angle of incidence $\phi_{0}$ and the thickness of gold layer $d_{g}$. According to the above equations, the reflected intensity of a $d_{p r}$ thick protein layer $I_{r}=I\left(\varphi_{0}, d_{g}, d_{p r}\right)$ could be simulated, so that the image contrast $\Delta I=I\left(\varphi_{0}, d_{g}, d_{p r}\right)-I\left(\varphi_{0}, d_{g}, 0\right)$ could be shown in Figure 6 . The absolute value of the maximum image contrast for a

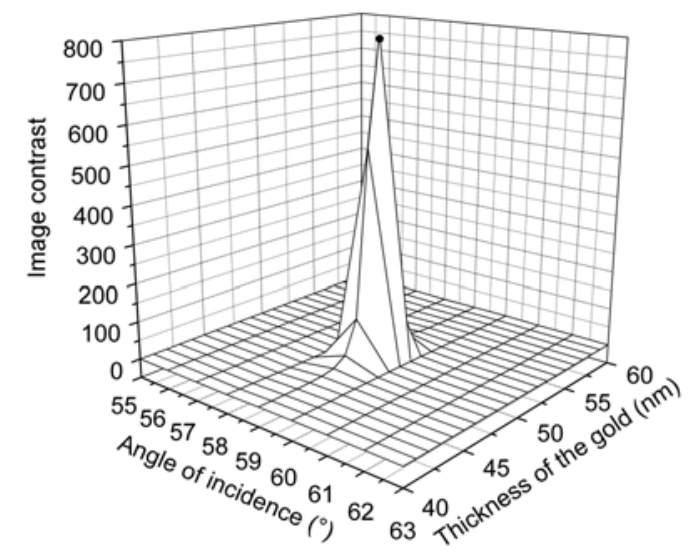

Figure 6 The image contrast of a $2 \mathrm{~nm}$ thick protein layer on the gold layer vs. the angle of incidence and the thickness of the gold layer. The refractive indices of the glass, the gold-layer, the protein layer and the liquid are $1.72,0.19+3.10 \mathrm{j}, 1.46$ and 1.33 at $633 \mathrm{~nm}$, respectively.
$2 \mathrm{~nm}$ thick protein layer is 814 which corresponds to $50 \mathrm{~nm}$ thickness of gold layer and the angle of incidence $59^{\circ}$, the point where the intensity of the evanescent wave reaches the maximum. The simulated result indicates the enhancement effect of the gold layer in image contrast is the same as that in the intensity of evanescent wave. The image contrast of the protein layer is enlarged by $10^{7}$ times, which makes the visualization of the protein layer possible.

\section{Optimized polarization settings for image contrast}

For the optimization of image contrast, the polarization state of light should be taken into account as shown above. Figure 7 shows the simulated optimized settings of the azimuth $A=178^{\circ}, P=35^{\circ}$ and $A=2^{\circ}, P=145^{\circ}$ equally, the angle of incidence $\varphi_{0}=59^{\circ}$ and the thickness of the gold $d_{g}=50 \mathrm{~nm}$. The maximum contrast of protein layer/substrate is 816.7 . The polarization of light just slightly improves the image contrast at the maximum.

\section{Discussion}

For the visualization of protein layer with an evanescent wave imaging system, the impact of the incident angle, the gold layer, and the polarization on the image contrast is theoretically simulated above. From Figures 3 and 5, we notice that the angle of incidence could be optimized for the maximum imaging contrast, at which a 100 times increase can be obtained with a polarization optimized setting. When the surface enhancement effect of a gold layer in Figure 6 is considered, an amazing increase of $10^{7}$ appears, but an interesting phenomenon is that the maximum values of image contrasts corresponding to the gold layer induced imaging

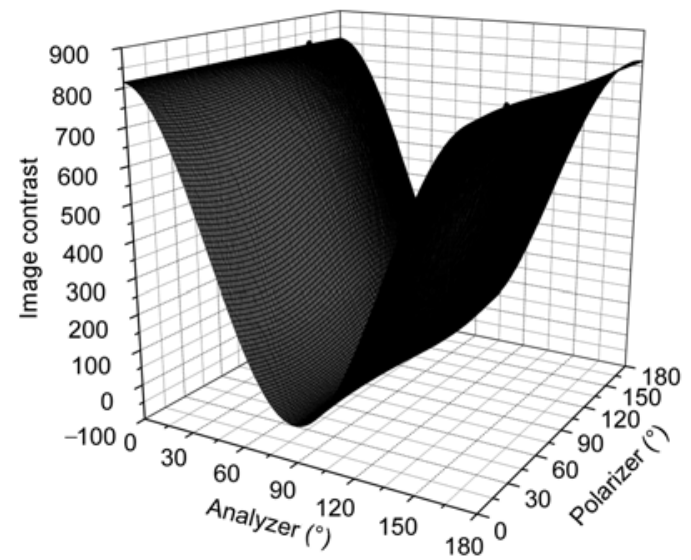

Figure 7 The image contrast vs. the azimuth setting of the polarizer and the analyzer within $0^{\circ}-180^{\circ}$. The sample is the glass/gold-layer/protein layer/liquid. The maximum contrast is 816.7 in the optimized settings of $A=178^{\circ}, P=35^{\circ}$ and $A=2^{\circ}, P=145^{\circ}$. 
system with and without the polarization optimization are almost the same. These results are much different from the imaging system without the gold layer. It seems that the gold layer results in a surface enhanced effect on the image contrast, and at the same time, in a depolarization effect. Here we should answer if a polarization optimization is still necessary or not.

From the observation above, we see that the image contrast is very sensitive to the thickness of the gold layer and critical, which should be well controlled. In fact, it is very sensitive not only to the thickness, but also to the refractive index of the gold layer, which we have not simulated here. Undoubtedly the image contrast is very sensitive to properties of the gold layer, or some metal layers used for the enhancement. In practice, the thickness and refractive index of gold layer can not be controlled as well as what we do in the simulation, so the measurement is not always performed under the optimized conditions. In this case, the real setting in experiments is in the neighboring region of the optimization. The image contrast without and with the polarization vs. the thickness of the gold in close proximity the optimized setting is shown in Figure 8(a). When the thickness of the gold is set at the optimized condition, the polarization optimization on the imaging contrast is indistinctive. That's because in the optimized setting of the incident angle and the thickness of the gold, the ellipsometric angle $\psi$ will reach a minimum value and $\Delta$ drops discontinuously a value.
It's a singular point for ellipsometric measurement just at which the ellipsometric measurement is invalid [9], so that the ellipsometric measurement always avoids the setting under the condition but it's sensitive nearby. Some effective comparison which is around the optimized thickness should be considered in Figures 8(b) and (c), where the polarization plays an important role in improving the image contrast.

Based on the theoretical analysis for settings of the imaging system, we have set up a similar experimental system to visualize an Immunoglobulin (IgG) adsorption layer. The following are the experimental conditions. The light source wavelength is $633 \mathrm{~nm}$ and the angle of incidence is set at $59^{\circ}$. The incident ambient is SF10 glass with a reflective index 1.72. An IgG layer with a thickness of $3.1 \mathrm{~nm}$ adsorbed directly on a SF10 glass plate which is imaging with a CCD camera can not be visualized in the solution of phosphate-buffered saline (PBS; pH7.4), nor can the polarization optimization settings of imaging system due to the low image contrast. Then, another sample is a $3.5 \mathrm{~nm}$ thick IgG layer adsorbed on a vacuum deposited gold layer with the thickness of $(37.5 \pm 0.8) \mathrm{nm}$ and the refractive index $N=0.19+3.10 \mathrm{j}$ and $2 \mathrm{~nm}$ thick Chromium interlayer on the SF10 glass. Note the thickness of gold layer is not the same as the optimized thickness since it's hardly obtained in practice. The evanescent wave imaging system with polarization is the conventional PCSA system [16] as shown in Figure 1. The imaging results are shown in Figure 9(a)
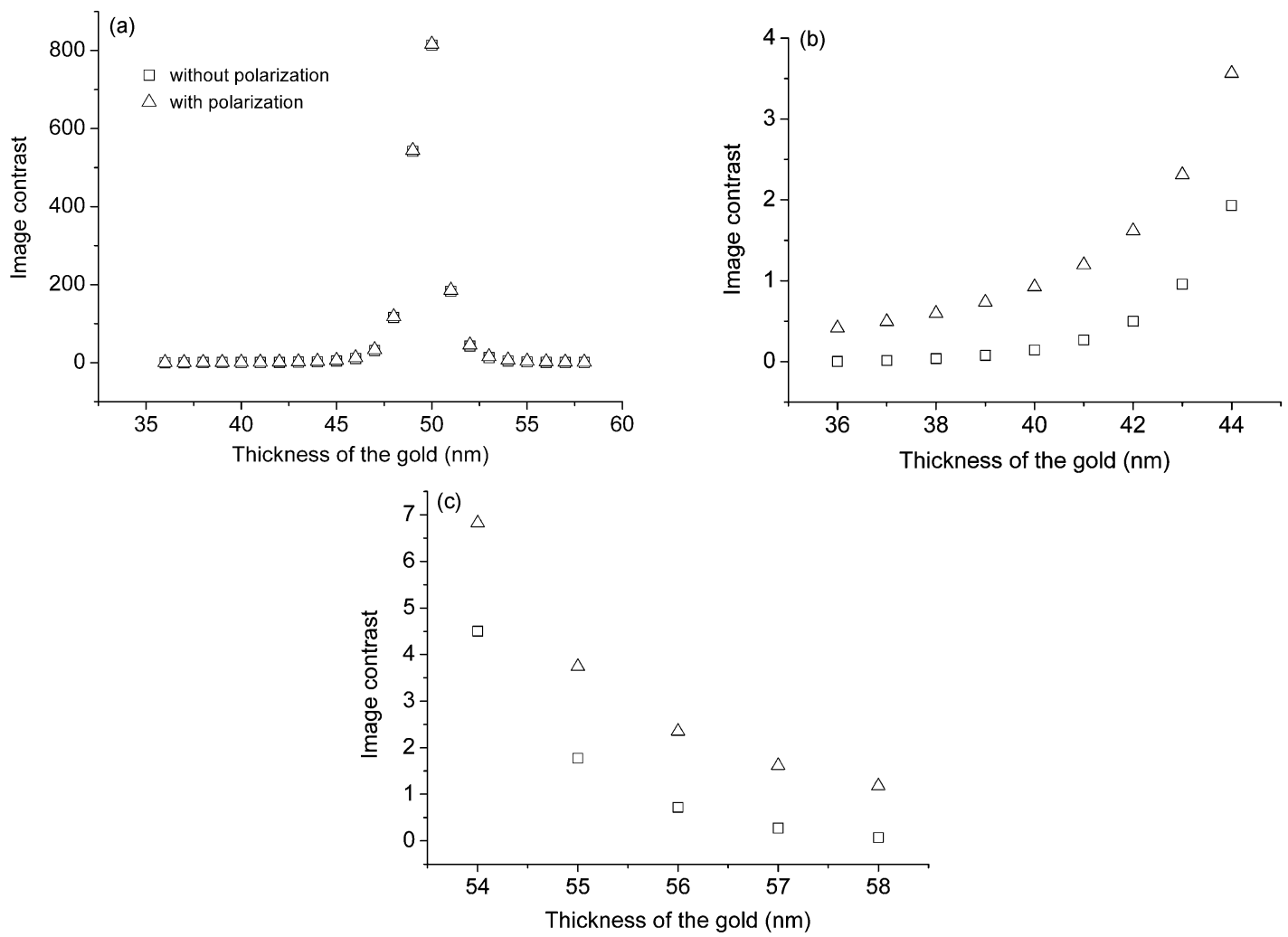

Figure 8 The image contrast of a $2 \mathrm{~nm}$ thick protein layer on the gold-layer vs. the thickness of the gold-layer in the imaging system with and without polarization setting. The " $\Delta$ " and the " $\square$ " represent the image contrast with and without polarization setting, respectively. 


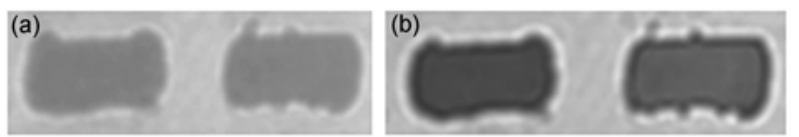

Figure 9 (a) The image of the substrate (left) and the IgG adsorption layer (right) by the imaging system without the polarization. (b) The image of the substrate (left) and the IgG adsorption layer (right) by the imaging system with an optimized polarization setting.

without polarization elements and in Figure 9(b) with polarization setting optimization. The former is obviously lower than the latter.

The contrast of the image shown in Figure 9 is measured. The normalized imaging contrast without and with the polarization imaging system is shown in Figure 10. The effect of the polarization is clear and the image contrast is improved about 14 times under the polarization optimization setting, which is close to the theoretical simulation result.

The gold layer plays an important role in improving the image contrast of protein adsorption layer visualization. Besides the effect of the gold thickness on the image contrast, the effect of the gold layer structure may impact it also. For example, a gold nano-particle layer raises a localized surface plasmons (LSPs) phenomenon, which brings an enhancement of electromagnetic wave at the interface [17] and may further improve the image contrast.

\section{Conclusion}

In order to visualize a protein adsorption layer with an evanescent wave imaging, a theoretical simulation has been made that the angle of incidence could be optimized for the maximum image contrast, at which a 100 times increase can be obtained with an optimized polarization setting for the sample system of glass/protein adsorption layer/liquid. The

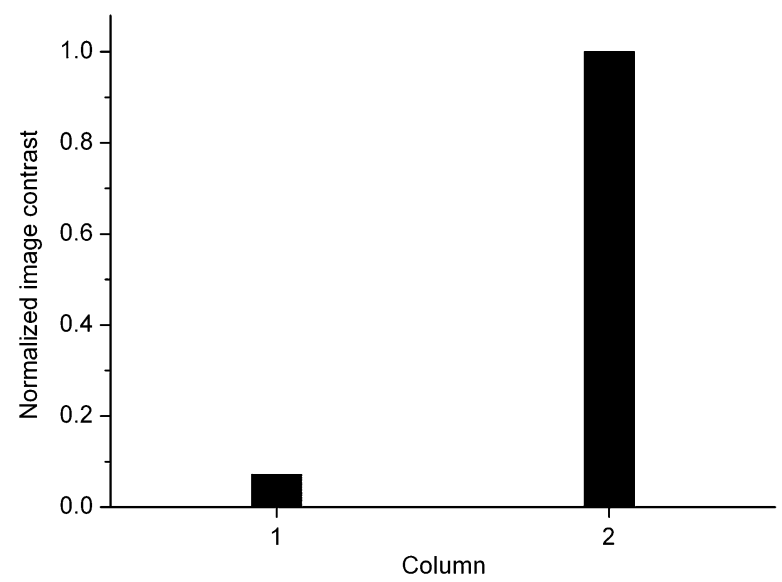

Figure 10 Column 1: Normalized image contrast without the polarization. Column 2: Normalized image contrast with the polarization. image contrast between a protein adsorption layer and substrate can be further improved about $10^{7}$ times by the surface enhancement effect of an optimized thickness of gold layer. In practice, a real sample system is not feasible with the thickness and refractive index optimization of gold layer and it is quite crucial and hardly to be reached, so experiments are performed under the optimized conditions. In this case, the polarization setting has improved the image contrast tremendously by experiments. The evanescent wave imaging provides an effectual approach to visualizing protein adsorption layers and maybe other similar sample systems. Even though the experimental conditions are out of the maximum optimization settings, the imaging system is still able to visualize a protein monolayer clearly and show a potential for biomolecule interaction measurements.

We gratefully acknowledge the Chinese Academy of Sciences, the National High Technology Research and Development Program of China (Grant No. 02Z4) and the National Basic Research Program of China (Grant No. 2009CB320302) for their supports.

1 Harrick J H. Internal Reflection Spectroscopy. Ossining, NY: Harrick Scientific Corporation, 1979

2 Novotny L, Hecht B. Principle of Nano-optics. Cambridge: Cambridge University Press, 2006

3 Prasad P N. Introduction to Biophotonics. New Jersey: John Wiley \& Sons, 2003

4 Sako Y, Shigeru M, Toshio Y. Single-molecule imaging of EGFR signalling on the surface of living cells. Nat Cell Biol, 2000, 2: $168-172$

5 Lagerholm B C, Weinreb G E, Jacobson K, et al. Detecting microdomains in intact cell membranes. Annu Rev Phys Chem, 2005, 56: 309-336

6 Rahn J R, Hallock R B. Antibody-binding to anti-gen-coated substrates studied with surface-plasmon oscillations. Langmuir, 1995, 11: $650-654$

7 Nilsson P, Persson B, Uhlen M, et al. Real-time monitoring of DNA manipulations using biosensor technology. Anal Biochem, 1995, 224: 400-408

8 Charles T C, Gibum K. SPR microscopy and its applications to high-throughput analyses of biomolecular binding events and their kinetics. Biomaterials, 2007, 28: 2380-2392

9 Azzam R M A, Bashara N M. Ellipsometry and Polarized Light. New York: North-Holland Pub. Co, 1977

10 Arwin H, Poksinski M, Johansen K. Total internal refection ellipsometry: Principle and applications. Appl Opt, 2004, 43: 3028-3036

11 Daniel A, Edward H H, Robert M F. Total inter-nal reflection fluorescence. Top Fluoresc Spectrosc, 1992, 3: 289-343

12 Arwin H. Optical properties of thin layers of bo-vine serum albumin, g-globulin, and Hemoglobin. Appl Spectrosc, 1986, 40: 313-318

13 Jin G, Jansson R, Arwin H. Imaging ellipsometry revisited: developments for visualization of thin transparent layers on silicon substrates. Rev Sci Instrum, 1996, 67: 2930-2936

14 Chen Y Y, Meng Y H, Jin G. Optimization of off-null ellipsometry for air/solid interfaces. Appl Opt, 2007, 46: 8475-8481

15 Nagata K, Handa H. Real-Time Analysis Of Biomolecular Interactions. Tokyo: Springer-Verlag, 2000

16 Chen Y Y, Wang Z H, Meng Y H, et al. Biosensor with total internal reflection imaging ellipsometry. Int J Nanotechnol, 2007, 4: 171-178

17 Hutter E, Fendler J H. Exploitation of localized surface plasmon resonance. Adv Mater, 2004, 16: 1685-1705 